\title{
T2 mapping evaluation of changes in cartilage matrix after PRP and HA injection therapy in knee osteoarthritis: a prospective, randomized, double blind, placebo controlled study
}

\section{Matjaz Vogrin}

Department of Orthopaedic Surgery, University Medical Center Maribor, Slovenia Jakob Naranda

Department of Orthopaedic Surgery, University Medical Center Maribor, Slovenia Mario Gorenjak

Faculty of Medicine, University Of Maribor, Slovenia

\section{Tomaz Bajec}

Department of Orthopaedic Surgery, University Medical Center Maribor, Slovenia

\section{Domen Mongus}

Faculty of Electrical Engineering and Computer Science, University of Maribor, Slovenia

\section{Robert Pintaric}

Department of Radiology, University Medical Center Maribor, Slovenia

\section{Igor Mijatovic}

Department of Orthopaedic Surgery, University Medical Center Maribor, Slovenia

Robi Kelc ( $\nabla$ robi.kelc@gmail.com )

Department of Orthopaedic Surgery, University Medical Center Maribor https://orcid.org/0000-00032758-3560

\section{Research article}

Keywords: osteoarthritis, cartilage degeneration, intra-articular therapies, platelet-rich plasma, viscosupplement, hyaluronic acid

Posted Date: June 19th, 2020

DOl: https://doi.org/10.21203/rs.3.rs-36493/v1

License: (9) This work is licensed under a Creative Commons Attribution 4.0 International License. Read Full License 


\section{Abstract}

\section{Background}

In this study we focused on evaluating the effect of intra-articular platelet-rich plasma (PRP) and hyaluronic acid (HA) on the qualitative and quantitative properties of the articular cartilage in patients with knee osteoarthritis (OA).

\section{Methods}

We evaluated 264 patients undergoing treatment of moderate knee OA. Patients aged between 40 and 70 years were included with articular K-L stage $\leq 2$. Patients were randomized into three groups and treated with three doses of intra-articular PRP, HA, or placebo injection. Patients were evaluated clinically and by using T2 mapping.

Results

Both PRP $\left(619.05 \pm 243 \mathrm{~ms} ; \mathrm{p}=2.7 \times 10^{-5}\right)$ and HA $\left(637.12 \pm 273 \mathrm{~ms} ; \mathrm{p}=1 . \times 10^{-4}\right)$ group showed statistically significant lower post-application $\mathrm{T} 2$ relaxation times in comparison to the placebo group (859.80 $\pm 406 \mathrm{~ms})$.

Cartilage thickness increased significantly after PRP $(2.41 \pm 0.87$ vs. $2.58 \pm 0.97 \mathrm{~mm}, \mathrm{p}=0.033)$ and HA $(2.28 \pm 0.81$ vs. $2.80 \pm 1.00 \mathrm{~mm}, \mathrm{p}=1.8 \times 10)$ applications. There was a significant decrease in WOMAC pain score by $30 \%(p=0,001)$ and the overall WOMAC score by $\left.27 p=1.9 \times 10^{-4} \%\right)$ in PRP group. The decrease in WOMAC pain score and the overall WOMAC score in the placebo group was similar to the results observed in the PRP group. In the HA group a significant decrease in WOMAC pain score by $14 \%$ $(p=0,005)$ was observed only at the first follow-up, whereas no difference was observed at the second follow-up.

Conclusions

T2 mapping of cartilage tissue may aid to monitor its properties after the intra-articular therapies in knee $\mathrm{OA}$. However, there is a discrepancy between clinical findings and the results of T2 mapping, suggesting questionable grounds for applying intra-articular therapy.

\section{Background}

Knee Osteoarthrosis (OA) is a clinically important syndrome presented with pain, functional limitations, and decreased life quality. Osteoarthrosis is pathomorphologically characterized by the degeneration and loss of cartilage, bone remodeling, subchondral sclerosis and inflammation [1]. Since knee OA is a progressive disease, it is treated symptomatically with physical therapy and non-steroid antiinflammatory drugs (NSADs) in early stages, followed by intraarticular injections of hyaluronic acid (HA) 
and platelet-rich plasma (PRP) in moderate cases; however, it can also lead to severe disability and joint replacement [2].

PRP is plasma prepared from patients' own blood and has a higher concentration of platelets than normal plasma. Platelets are applied intraarticularly with injection. Growth factors are released from platelets and after stimulation are said to modulate biological processes such as cartilage healing and inflammation, and consequently improve the symptoms of OA [3].

$\mathrm{HA}$ is a polysaccharide compound containing glucuronic acid and acetyl glucosamine. As the concentration and molecular weight of hyaluronic acid are reduced in OA, intraarticular injections of HA are a therapeutic option. HA provides viscoelasticity of synovial fluid and stimulates the formation of endogenous hyaluronic acid $[4,5]$. In addition, HA can be also effective with the stimulation and accumulation of proteoglycan, inhibition of inflammatory mediators and analgesic effect $[5,6]$.

The effects of intraarticular applications of HA and PRP has been studied in multiple trials in symptomatic OA patients with a focus on the knee function, pain, and quality of life [7, 8]; however, all these trials provide limited data based on qualitative and quantitative changes of articular cartilage after therapeutic applications.

In this randomized, double-blind, placebo-controlled study, we evaluated the impact of intraarticular administration of PRP and HA in patients with knee OA. Our research was mainly focused T2 mapping, the quantitative biochemical MRI technique, proven sensitive in measuring biomechanical and biochemical properties of cartilage such as collagen, glycosaminoglycan and water content and collagen orientation $[9,10]$. The usefulness of T2 mapping was already demonstrated in different clinical settings e.g. in the diagnosis of early cartilage degeneration $[11,12]$, in the effect of loading on articular cartilage [13], in pre-surgical planning [14] and in the evaluation of successful treatment of cartilage lesions [1517]. Focal increases in $T 2$ relaxation times within cartilage have been associated with matrix damage, loss of collagen integrity and an increase in water content $[9,10,18]$. To the best of our knowledge, this is the first study that applies the T2 imaging to evaluate the efficacy of intraarticular application of PRP and HA in OA knee.

\section{Methods}

Patients (selection, inclusion, intervention)

The prospective double-blind parallel randomized controlled trial was approved by the institutional ethical committee. 264 patients were randomly assigned into three groups (intra-articular placebo, PRP, and HA injection) and evaluated at 0,2 and 6 months of follow-up. The inclusion and exclusion criteria are listed in Table 1. Patients were prospectively evaluated at 0,2 and 6 moths of follow-up using the WOMAC score. Additionally, range of motion (ROM) and trans-patellar circumference of both knees was measured and patient satisfaction as well as any adverse event were recorded. To ensure the blinding, all patients enrolled in the study underwent blood harvesting in order to obtain PRP for further knee injections 
performed only in one third of them following the randomized selection. The randomized list of all patients was provided by an independent statistician and the injection solution (PRP, HA or saline solution) was assigned to the patients just before the application itself. Three injection applications were performed at weekly intervals. In addition, all clinical evaluations were performed by an independent medical member to keep the study double blinded.

Table 1

Inclusion and exclusion criteria

\begin{tabular}{|l|}
\hline Inclusion criteria \\
\hline $40-70$ years old \\
\hline History of knee pain and/or swelling in last 4 months \\
\hline Kallgren, Lawrence score 2 or 3 \\
\hline Exclusion criteria \\
\hline Older than 70 years \\
\hline Kallgren, Lawrence higher than 3 \\
\hline Systemic disease \\
\hline BMI $>35$ \\
\hline Hematologic conditions (anemia, coagulopathies) \\
\hline Inflammatory disease \\
\hline Immunosuppressive therapy \\
\hline Axial knee deformity (valgus/varus $>5$ degrees) \\
\hline Allergies \\
\hline
\end{tabular}

PRP was prepared using Arthrex ACP kit (Arthrex, Naples, US). $15 \mathrm{~mL}$ of venous blood was drawn and then centrifuged at $1500 \mathrm{rpm}$ for 10 minutes. Plasma was removed using a double syringe system, leaving the final product $5 \mathrm{~mL}$ of PRP. Anticoagulants were not used as PRP was utilized within 15 minutes.

In the HA group, Suplasyn (Mylan) was used at 3 weekly intervals, each $2 \mathrm{~mL}$ syringe containing $20 \mathrm{~mL}$ of sodium hyaluronate with molecular weight of 500-730 kDa.

Outcome measures

Questionnaire and MRI-based findings were used as outcome measures. Our primary outcome measure was qualitative assessment of cartilage using T2 maps, and the secondary outcome measure the change in cartilage thickness 6 months after the applications. Tertiary outcomes were pain as a subscore of 
WOMAC and overall WOMAC score. The WOMAC scoring was performed at three different time points: (1) before applications, (2) 2 months after the last application and (3) 6 months after the last application.

MRI protocol

Of the initial 264 patients, 204 of them performed both MRI examinations, the first before the application and the second 6 months after the last application. All MRI examinations were performed using 3-Tesla MRI (General Electrics Signa Excite xt) using an eight-channel knee coil. Coil and system QA were performed daily prior to any examination. Knee joint was fixed at $15^{\circ}$ flexion. The MR images of T 2 mapping and 3D gradient-echo sequences with pre-pulse fat-saturation (3DGRE FAT-SAT) were obtained. Before carrying out the investigation, all patients performed a 10-minute walk in the hall after initial 30 minute rest to control the pre-loading of cartilage.

MRI analysis

To achieve adequate comparison of pre- and post-application MR images, two images were aligned by specially designed software. Methodology, used in this study, consisted of image registration, definition of cross-section, and extraction of image intensity profiles [19]. During image registration, two images are transformed by aligning their resolution, position, and rotation in order to achieve spatial matching of key points, defined by distinct image features like sharp edges. Such alignment allows for direct comparison of pre-application and post-application MR images (ROI positioning) and comparison of spatial frequencies of intensity values in arbitrary direction for purpose of cartilage thickness assessment.

All MRI evaluations were performed by a blinded musculoskeletal radiologist. Functool application was used on an ADW 4.4 workstation to make measurements on T2 quantitative mappings. Articular cartilage evaluations were performed on regions of interests (ROIs) set on the medial femoral weight bearing condyle on an area directly above the anterior meniscus horn in sagittal slice. The cartilage was divided into two zones according to its thickness, one being closer to the bony side (ROI 1) and the second being closer to the articular side (ROI 2) as shown in Fig. 1.

3DGRE FAT-SAT pulse rate was used for cartilage thickness measurements. $1.8 \mathrm{~mm}$ slice depth was used with $0.9 \mathrm{~mm}$ layover, thus enabling 3D visualization of cartilage. After aligning two images (preapplication and post-application), as described above, a user-defined line of cartilage cross-section was rasterized [19] and intensity values of obtained voxels from multiple images plotted on a single graph for comparison, as seen in Fig. 2. Finally, pixels were calculated into millimeters to express the cartilage thickness.

\section{Statistical analysis}

Data were analyzed using IBM SPSS Statistics 25.0 (IBM Inc., Armonk, New York, USA), R 3.5.3 (www.rproject.org, R Core Team (2017), Vienna, Austria) and GPower 3.1 (G*Power, Kiel, Germany). Comparisons of continuous variables across nominal groups were carried out using Mann-Whitney U-Test or pairwise 
Kruskal-Wallis H-Test after Kolmogorov-Smirnov test of normality. Paired samples were analyzed using the Wilcoxon signed-rank test. Statistical power was calculated by means of post hoc Wilcoxon-MannWhitney test or Wilcoxon signed-rank test for paired samples. Effect sizes were determined for each statistical analysis separately. The Spearman's correlation between paired groups was determined where needed. Alpha was set to $5 \%(a=0.05)$. P value $<0.05$ and Power $>80 \%$ was considered statistically significant.

\section{Results}

Qualitative cartilage assessment: T2 mapping

Baseline characteristic between groups were similar as there were no statistically significant differences between them. The overall mean values of $\mathrm{T} 2$ relaxation times of $\mathrm{ROI} 1$ and $\mathrm{ROI} 2$ before applications are listed in Table 2.

Table 2

ROI 1 and ROI 2 baseline values across groups

\begin{tabular}{|lllll|}
\hline & Control $(\boldsymbol{n}=\mathbf{5 1})$ & PRP $(\boldsymbol{n}=\mathbf{7 5})$ & HA $(\boldsymbol{n}=\mathbf{7 8})$ & P value \\
\hline ROI 1 & $603 \pm 198$ & $548 \pm 255$ & $545 \pm 186$ & 0.090 \\
\hline ROI 2 & $711 \pm 270$ & $756 \pm 324$ & $722 \pm 302$ & 0.905 \\
\hline
\end{tabular}

There were no statistically significant differences between pre- and post-application in ROI 1 relaxation times in any of the groups. However, some differences were noted in ROI 1 post-application between PRP and placebo ( $502.87 \pm 189$ vs. $668.84 \pm 278, p=0.005$, power $=96 \%$ ) and between the HA and placebo group (531.18 \pm 219 vs. $668.84 \pm 278, p=0.026$, power $=83 \%$ ), and no statistically significant differences between PRP and HA (Fig. 3). In the PRP group, a significant decrease in T2 relaxation times in ROI 2 was observed 6 months after the application $\left(619 \pm 243 ; p=2.1 \times 10^{-4}\right.$, power $\left.=100 \%\right)$ when compared to the pre-application (756.14 \pm 323 ). A similar statistically suggestive decrease was observed in the HA group $(637.12 \pm 273$ vs. $722.14 \pm 302, p=0.002$, power $=56 \%)$. On the contrary, a statistically suggestive increase in post-application ROI 2 relaxation times was observed in the placebo group ( $859.80 \pm 406 \mathrm{vs}$. $711.08 \pm 269, p=0.029$, power $=72 \%$ ). Subsequently, a comparison of post-application T2 ROI 2 relaxation times was performed between the groups. Both, $\operatorname{PRP}\left(619.05 \pm 243 ; p=2.7 \times 10^{-5}\right.$, power $=$ $97 \%)$ and $\mathrm{HA}\left(637.12 \pm 273 ; \mathrm{p}=1 . \times 10^{-4}\right.$, power $\left.=93 \%\right)$ groups showed statistically significant lower ROI2 post-application relaxation times in comparison to the placebo $(859.80 \pm 406)$ post-application relaxation times.

Assessment of cartilage morphology: thickness

No statistically significant difference was observed in cartilage thickness between groups at preapplication $(p=0.352)$. The evaluation of cartilage thickness pre- vs. post-application showed no 
statistically significant difference in the placebo group (Fig. 4). On the other hand, a tendency for increase in cartilage thickness was observed 6 months after the application in the PRP group ( $2.41 \pm 0.87$ vs. 2.58 $\pm 0.97, p=0.033$, power $=55 \%$ ), whereas in the HA group, a statistically significant increase of cartilage thickness was observed $\left(2.80 \pm 1.00, p=1.8 \times 10^{-5}\right.$, power $\left.=100 \%\right)$ when compared to pre-application $(2.28 \pm 0.81)$.

WOMAC: pain and overall score

Baseline characteristic between the groups were similar as there were no statistically significant differences between them. The overall mean values are listed in Table 3. There were no statistically significant differences between the groups at any time point observed; however, we noticed significant changes between time points within each group. In the PRP group, a significant decrease in WOMAC pain score was observed in both 2 months after $\left(7.36 \pm 4.21 ; p=2.1 \times 10^{-5}\right.$, power $\left.=99.8 \%\right)$ and 6 months after the applications $(6.50 \pm 4.30 ; p=0.001$, power $=100 \%)$ when compared to pre-application $(9.37 \pm 3.87)$ (Fig. 5). Similarly, a decrease in overall WOMAC score was observed both after the first (44.46 $\pm 19.06 ; p=$ $2.3 \times 10^{-5}$, power $\left.=96.4 \%\right)$ and second post-application visit $\left(36.35 \pm 21.04 ; p=1.9 \times 10^{-4}\right.$, power $\left.=100 \%\right)$ when compared to pre-application (50.04 \pm 19.52$)$ (Fig. 6).

Table 3

Baseline values across groups

\begin{tabular}{|lllll|}
\hline & Control $(\mathbf{n}=\mathbf{6 6})$ & PRP $(\boldsymbol{n}=\mathbf{8 7})$ & HA $(\mathbf{n}=\mathbf{8 7})$ & P value \\
\hline WOMAC pain & $9.55 \pm 3.53$ & $9.37 \pm 3.9$ & $8.86 \pm 3.72$ & 0.575 \\
WOMAC overall & $50.45 \pm 18.92$ & $50.04 \pm 19.52$ & $48.24 \pm 16.44$ & 0.552 \\
\hline
\end{tabular}

In placebo group a similar trend was observed. Statistically significant decrease of WOMAC pain score was observed 2 months after $(7.80 \pm 3.43 ; p=0.001$, power $=98.45 \%)$ and 6 months after the application $\left(7.06 \pm 4.39 ; p=5.3 \times 10^{-5}\right.$, power $\left.=100 \%\right)$ when compared to pre-application $(9.55 \pm 3.53)$. Similar, a decrease in overall WOMAC score was observed, but only after the second post-application visit (39.29 \pm 20.77; $p=0.001$, power $=100 \%)$ in comparison to pre-application $(50.45 \pm 18.92)$.

In the HA group, a statistically significant decrease in WOMAC pain score was observed 2 months after the application $(7.61 \pm 4.01 ; p=0.005$, power $=88.9 \%)$ when compared to pre-application $(8.86 \pm 3.72)$. A decrease trend in WOMAC pain score was also observed 6 months after application $(8.21 \pm 3.95 ; p=$ 0.008 , power $=47 \%$ ) when compared to pre-application $(8.86 \pm 3.72)$, but was not considered statistically significant due to low power. A statistically significant decrease was also observed for overall WOMAC score after first application $\left(40.50 \pm 18.72 ; p=7.9 \times 10^{-5}\right.$, power $\left.=99.89 \%\right)$ when compared to preapplication (48.24 \pm 16.44 ). At the second post-application visit, only suggestive statistical significance was observed $(43.67 \pm 18.42 ; p=0.016$, power $=77.73 \%)$.

\section{Discussion}


Quantitative MRI T2 mapping techniques have been used as a non-invasive biomarker of early degenerative changes in knee OA $[11,12,20]$. MRI relaxation times are translated into quantitative values of tissues [21] in order to show subtle changes in cartilage composition in the early stages [9, 10]. In this randomized, double-blind, placebo-controlled study T2 mapping was used specifically to detect any changes in the cartilage tissue after intraarticular treatment with PRP and HA in patients with knee OA. Specially designed software and methodology was utilized in order to generate T2 relaxation times in the region of interest (ROI) [19]. Since research in this field has mainly focused on the clinical outcome by monitoring various subjective questionnaires and clinical measurements [7, 8, 22] the T2 mapping represents a novelty in this research field.

The most important finding in the present study was a substantial focal decrease in T2 relaxation time after HA applications $(p=0.002$, power $=56 \%$ ) and a statistically significant decrease after PRP applications in patients with knee OA. The focal change in T2 relaxation times within each group was observed at the articular side of the cartilage (ROI 2), whereas there were no differences observed in the subchondral cartilage layer (ROI 1). These results indirectly suggest that the target location of intraarticular application is mainly on the articular side of the cartilage. On the contrary, T2 relaxation times in placebo group substantially increased $(p=0.029$, power $=72 \%)$. There were no differences in cartilage thickness at follow-up in the placebo group, whereas the thickness of articular cartilage substantially increased in PRP group $(p=0.033$, power $=55 \%)$ and significantly increased in HA group. This may in turn indicate a modification in the cartilage microstructure, collagen orientation, and water content after intraarticular PRP and HA applications $(5,6)$, possibly to prevent further deterioration of OA by restoring cartilage tissue $[9,10,18]$. Furthermore, T2 relaxation times were significantly lower in both ROI 1 and 2 after PRP and HA applications compared to the placebo group at follow-up. Therefore, PRP and HA applications might provide some sort of protective effect even in the deeper cartilage layer.

On the other hand, the differences in T2 relaxation times might be due to the altered loading of articular cartilage as a consequence of intraarticular applications [13]. Different volumes of intraarticular applications ( $5 \mathrm{ml}$ in PRP and $2 \mathrm{ml}$ in $\mathrm{HA}$ ) and extraarticular (subcutaneous) administration of saline solution in placebo group might have influenced the results of T2 relaxation times. A similar impact on T2 relaxation times were demonstrated in surgical treatments of cartilage lesions, especially in matrix autologous chondrocyte implantation (MACI) $[16,17]$. Since this is the first study using T2 mapping to evaluate a potential cartilage repair after intraarticular administration of PRP or HA, there is a lack of evidence regarding the interpretation of the T2 relaxation time of potentially "recovered" cartilage. Nevertheless, several parameters clearly indicate that there were some favourable changes in articular cartilage after intraarticular applications of HA and PRP compared to the placebo group at follow-up. Other factors that may affect T2 relaxation times, such as gender and age [12, 23, 24], BMI and daily activity [25], and time after loading $[13,26]$ were excluded by providing homogenous groups and using the pre-loading protocol.

The other objective of this study was to evaluate the effect of intraarticular applications of PRP and HA on knee function, pain, and quality of life by using WOMAC questionnaires and clinical examination. The 
positive effect of intraarticular applications in treatment of symptomatic knee OA was well documented in the literature $[7,8,22]$. The results of this study showed significant decrease in WOMAC pain score and overall WOMAC score in the PRP group at follow-ups, which is consistent with the literature [27, 28]. This, in combination with the results of T2 mapping, seems to be in favour of improving cartilage properties $(5,6)$. On the other hand, the results in the placebo group are surprising as the significant decrease in WOMAC scores was similar to the PRP group despite the deterioration of cartilage characteristics based on T2 mapping $[9,10,18]$. The opposite proved to be the case for the HA group since a significant decrease in WOMAC pain score was observed only at the first follow-up at 2 months, whereas no difference was observed at the second follow-up at 6 months. Similarly, mixed clinical outcomes were demonstrated in randomized controlled trials when the effect of intraarticular HA administration was compared to PRP in knee OA treatment $[2,29,30]$.

Finally, the results of T2 mapping seem to match the clinical findings and patients' satisfaction only in the PRP group, whereas this was not observed in the HA or placebo group. Namely, patients in the placebo group showed the same level of satisfaction (according to WOMAC score) compared to the PRP group, while in the HA group the level of satisfaction no longer improved over time. Thus, the actual significance of the observed changes in cartilage T2 mapping is questionable since it does not match the clinical findings. Although the results of T2 mapping indicated improving cartilage properties after receiving PRP or HA, there was no overall superior clinical improvement compared to the placebo group. Therefore, further research needs to be conducted in order to successfully validate the actual relevance of T2 mapping after administration of intraarticular therapy, possibly with concurrent histological analysis and clinical data. In addition, the long-lasting effect of $T 2$ changes and overall cost benefit ratio of intraarticular therapy in knee OA should also be re-evaluated.

In conclusion, T2 mapping of cartilage tissue may aid in monitoring cartilage improvement or deterioration after the administration of intraarticular therapy in knee OA. However, clinical data seem not to match the results of T2 mapping in all cases. Therefore, the actual significance of T2 mapping in this clinical setting should be further investigated. Intraarticular therapy did not demonstrate any superior effect on patient's satisfaction and level of pain compared to placebo group. Thus, the effectiveness of intraarticular therapy with PRP or HA in knee OA needs to be reassessed in placebo-controlled trials.

\section{Declarations}

Ethics approval and consent to participate

Th study was approved by the ethic committee of University Medical Center Maribor, Slovenia (Ref. Nr. IRP-2016/02-05)

Consent for publication

Not applicable 
Availability of data and materials

The datasets used and/or analysed during the current study are available from the corresponding author on request

Competing interest

None

Funding

None

Authors' contributions

MV - conception, study design, data collection, article drafting, critical revision of article, final approval of article to be published

$\mathrm{JN}$ - data interpretation, article drafting

TB - data collection

MG - data analysis and interpretation

DM - software developmentment, data analysis

$\mathrm{RP}$ - data collection

IM - data collection

RK - data interpretation, article drafting

Acknowledgements

None

\section{References}

1. Hunter DJ, Bierma-Zeinstra S, Osteoarthritis. Lancet. 2019;393:1745-59.

2. Han Y, Huang H, Pan J, Lin J, Zeng L, Liang G, et al. Meta-analysis Comparing Platelet-Rich Plasma vs Hyaluronic Acid Injection in Patients with Knee Osteoarthritis. Pain Med. 2019;20:1418-29.

3. Dhillon MS, Patel S, John R. PRP in OA knee - update, current confusions and future options. SICOT J. 2017;3:27. 
4. Altman RD, Manjoo A, Fierlinger A, Niazi F, Nicholls M. The mechanism of action for hyaluronic acid treatment in the osteoarthritic knee: a systematic review. BMC Musculoskelet Disord. 2015;16:321.

5. McArthur BA, Dy CJ, Fabricant PD, Valle AGD. Long term safety, efficacy, and patient acceptability of hyaluronic acid injection in patients with painful osteoarthritis of the knee. Patient Prefer Adherence. 2012;6:905-10.

6. Montañez-Heredia E, Irízar S, Huertas PJ, Otero E, Del Valle M, Prat I, et al. Intra-Articular Injections of Platelet-Rich Plasma versus Hyaluronic Acid in the Treatment of Osteoarthritic Knee Pain: A Randomized Clinical Trial in the Context of the Spanish National Health Care System. Int J Mol Sci. 2016;17.

7. Ong KL, Anderson AF, Niazi F, Fierlinger AL, Kurtz SM, Altman RD. Hyaluronic Acid Injections in Medicare Knee Osteoarthritis Patients Are Associated With Longer Time to Knee Arthroplasty. J Arthroplasty. 2016;31:1667-73.

8. Rayegani SM, Raeissadat SA, Taheri MS, Babaee M, Bahrami MH, Eliaspour D, et al. Does intra articular platelet rich plasma injection improve function, pain and quality of life in patients with osteoarthritis of the knee? A randomized clinical trial. Orthop Rev (Pavia). 2014;6:5405.

9. Mosher TJ, Dardzinski BJ. Cartilage. MRI T2 relaxation time mapping: overview and applications. Semin Musculoskelet Radiol. 2004;8:355-68.

10. Kim T, Min B-H, Yoon S-H, Kim H, Park S, Lee HY, et al. An in vitro comparative study of T2 and T2* mappings of human articular cartilage at 3-Tesla MRI using histology as the standard of reference. Skeletal Radiol. 2014;43:947-54.

11. Apprich S, Mamisch TC, Welsch GH, Stelzeneder D, Albers C, Totzke U, et al. Quantitative T2 mapping of the patella at 3.0T is sensitive to early cartilage degeneration, but also to loading of the knee. Eur J Radiol. 2012;81:e438-43.

12. Waldenmeier L, Evers C, Uder M, Janka R, Hennig FF, Pachowsky ML, et al. Using Cartilage MRI T2Mapping to Analyze Early Cartilage Degeneration in the Knee Joint of Young Professional Soccer Players. Cartilage. 2019;10:288-98.

13. Mamisch T, Trattnig S, Quirbach S, Marlovits S, White L, Welsch G. Quantitative T2 Mapping of Knee Cartilage: Differentiation of Healthy Control Cartilage and Cartilage Repair Tissue in the Knee with Unloading-Initial Results. Radiology. 2010;254:818-26.

14. Nishioka H, Hirose J, Nakamura E, Okamoto N, Karasugi T, Taniwaki T, et al. Detecting ICRS grade 1 cartilage lesions in anterior cruciate ligament injury using T1 $\rho$ and T2 mapping. Eur J Radiol. 2013;82:1499-505.

15. Holtzman DJ, Theologis AA, Carballido-Gamio J, Majumdar S, Li X, Benjamin C. T(1 $\rho)$ and T(2) quantitative magnetic resonance imaging analysis of cartilage regeneration following microfracture and mosaicplasty cartilage resurfacing procedures. J Magn Reson Imaging. 2010;32:914-23.

16. Olivos Meza A, Cortés González S, Ferniza Garza JJ, Pérez Jiménez FJ, Enrique VC, Ibarra C. Arthroscopic Treatment of Patellar and Trochlear Cartilage Lesions with Matrix Encapsulated 
Chondrocyte Implantation versus Microfracture: Quantitative Assessment with MRI T2-Mapping and MOCART at 4-Year Follow-up. Cartilage. 2019;1947603519835909.

17. Correa Bellido P, Wadhwani J, Gil Monzo E. Matrix-induced autologous chondrocyte implantation grafting in osteochondral lesions of the talus: Evaluation of cartilage repair using T2 mapping. $J$ Orthop. 2019;16:500-3.

18. Hannila I, Lammentausta E, Tervonen $\mathrm{O}$, Nieminen M. The repeatability of T2 relaxation time measurement of human knee articular cartilage. 28. New York: Magma; 2015.

19. Liu YK, Wang PJ, Zhao DD, Spelic D, Mongus D, Zalik B. Pixel-Level Algorithms for Drawing Curves. Warwick, UK; 2011. p. 33-40.

20. Baum T, Joseph GB, Karampinos DC, Jungmann PM, Link TM, Bauer JS. Cartilage and meniscal T2 relaxation time as non-invasive biomarker for knee osteoarthritis and cartilage repair procedures. Osteoarthritis Cartilage. 2013;21:1474-84.

21. Kester BS, Carpenter PM, Yu HJ, Nozaki T, Kaneko Y, Yoshioka H, et al. T1 $/$ /T2 mapping and histopathology of degenerative cartilage in advanced knee osteoarthritis. World J Orthop. 2017;8:350-6.

22. Di Martino A, Di Matteo B, Papio T, Tentoni F, Selleri F, Cenacchi A, et al. Platelet-Rich Plasma Versus Hyaluronic Acid Injections for the Treatment of Knee Osteoarthritis: Results at 5 Years of a DoubleBlind, Randomized Controlled Trial. Am J Sports Med. 2019;47:347-54.

23. Mosher TJ, Collins CM, Smith HE, Moser LE, Sivarajah RT, Dardzinski BJ, et al. Effect of gender on in vivo cartilage magnetic resonance imaging T2 mapping. J Magn Reson Imaging. 2004;19:323-8.

24. Kim HK, Shiraj S, Anton CG, Horn PS, Dardzinski BJ. Age and sex dependency of cartilage T2 relaxation time mapping in MRI of children and adolescents. AJR Am J Roentgenol. 2014;202:62632.

25. Matcuk GR, Jones IA, McIntyre JA, Burt R, Hwang D, Cen S, et al. Evaluation of Knee Cartilage Diurnal, Activity, and BMI-Related Variations Using Quantitative T2 Mapping MRI and Fitbit Activity Tracking. J Knee Surg. 2019.

26. Liess C, Lüsse S, Karger N, Heller M, Glüer C-C. Detection of changes in cartilage water content using MRI T2-mapping in vivo. Osteoarthr Cartil. 2002;10:907-13.

27. Lin K-Y, Yang C-C, Hsu C-J, Yeh M-L, Renn J-H. Intra-articular Injection of Platelet-Rich Plasma Is Superior to Hyaluronic Acid or Saline Solution in the Treatment of Mild to Moderate Knee Osteoarthritis: A Randomized, Double-Blind, Triple-Parallel, Placebo-Controlled Clinical Trial. Arthroscopy. 2019;35:106-17.

28. Görmeli G, Görmeli CA, Ataoglu B, Çolak C, Aslantürk O, Ertem K. Multiple PRP injections are more effective than single injections and hyaluronic acid in knees with early osteoarthritis: a randomized, double-blind, placebo-controlled trial. Knee Surg Sports Traumatol Arthrosc. 2017;25:958-65.

29. Cole BJ, Karas V, Hussey K, Pilz K, Fortier LA. Hyaluronic Acid Versus Platelet-Rich Plasma: A Prospective, Double-Blind Randomized Controlled Trial Comparing Clinical Outcomes and Effects on Intra-articular Biology for the Treatment of Knee Osteoarthritis. Am J Sports Med. 2017;45:339-46. 
30. Meheux CJ, McCulloch PC, Lintner DM, Varner KE, Harris JD. Efficacy of Intra-articular Platelet-Rich Plasma Injections in Knee Osteoarthritis: A Systematic Review. Arthroscopy. 2016;32:495-505.

\section{Figures}
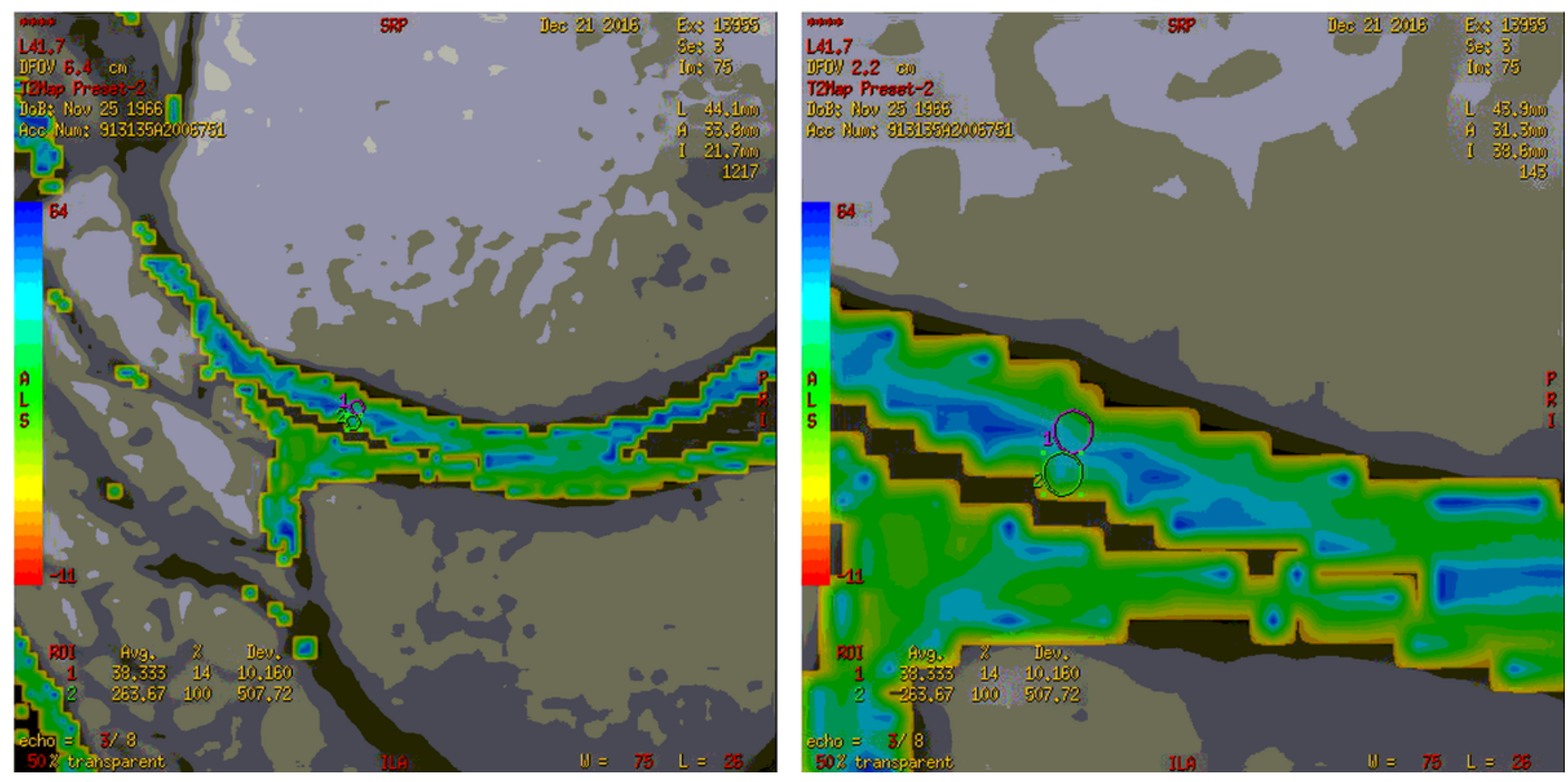

\section{Figure 1}

T2 mapping of articular cartilage. ROI 1 (deep layer, purple circle) and ROI 2 (superficial layer, green circle) set on the medial femoral condyle at the are above the anterior meniscus horn. 

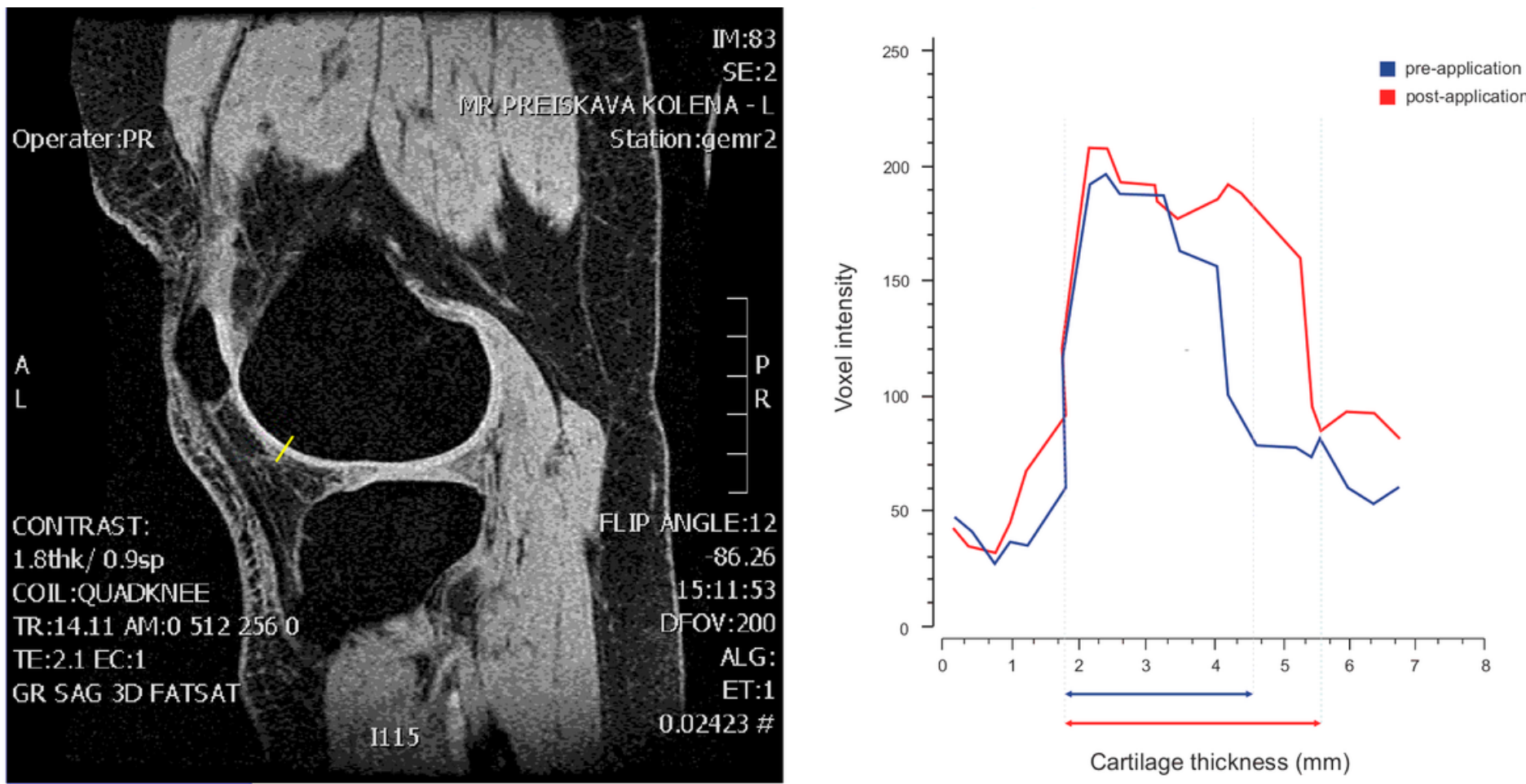

Figure 2

Articular cartilage thickness. After aligning pre- and post-application images, a line over the cartilage was defined, the cross-section rasterized and intensity values of voxels plotted on a graph for comparison. A sharp increase in intensity defines the edge of the cartilage (next to subchondral bone), whereas the drop represents its edge of the cartilage.
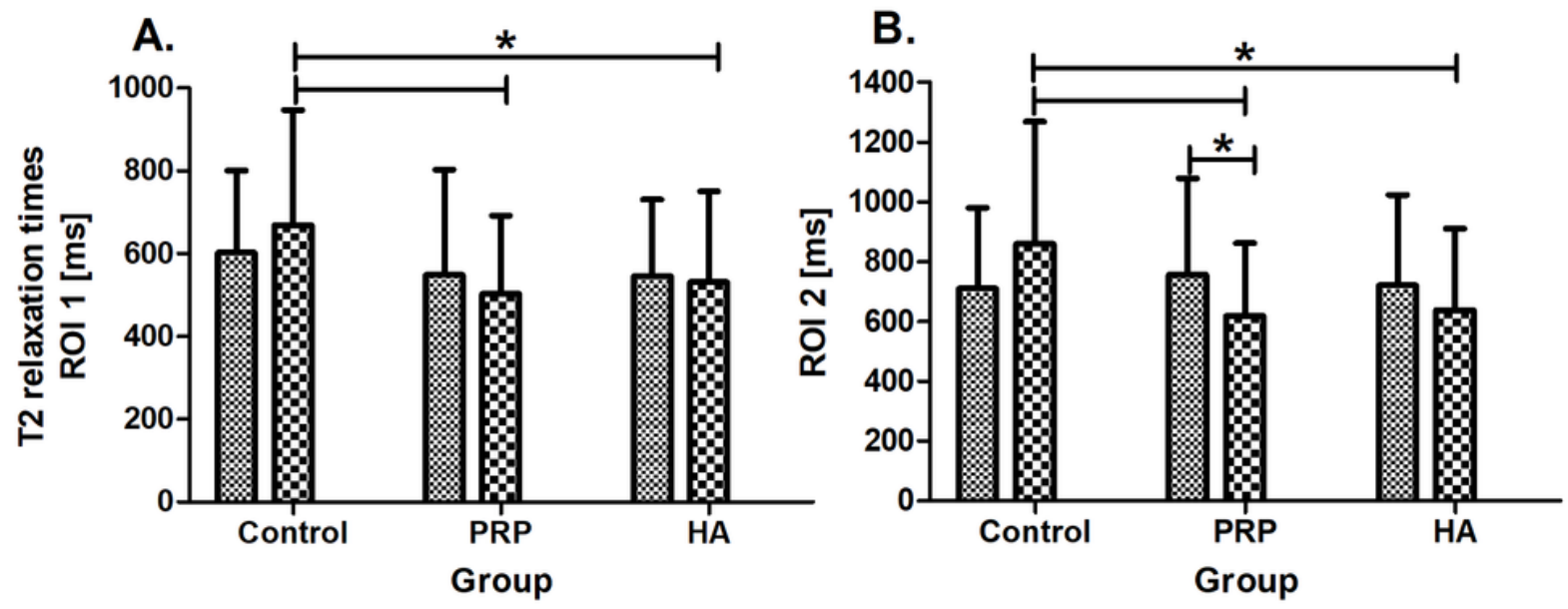

Pre-application

$\mathbf{E}$ Post-application

Figure 3

Comparison of T2 relaxation times at ROI 1 and ROI 2 between groups before the application and 6 months after the application. Data are presented as mean values $\pm S D$. Statistically significant differences between pre- and post-application were determined using the Wilcoxon signed-rank test and in comparison, for control the Mann-Whitney U-test ( $\left.{ }^{*} \mathrm{P} \leq 0.05\right)$. 


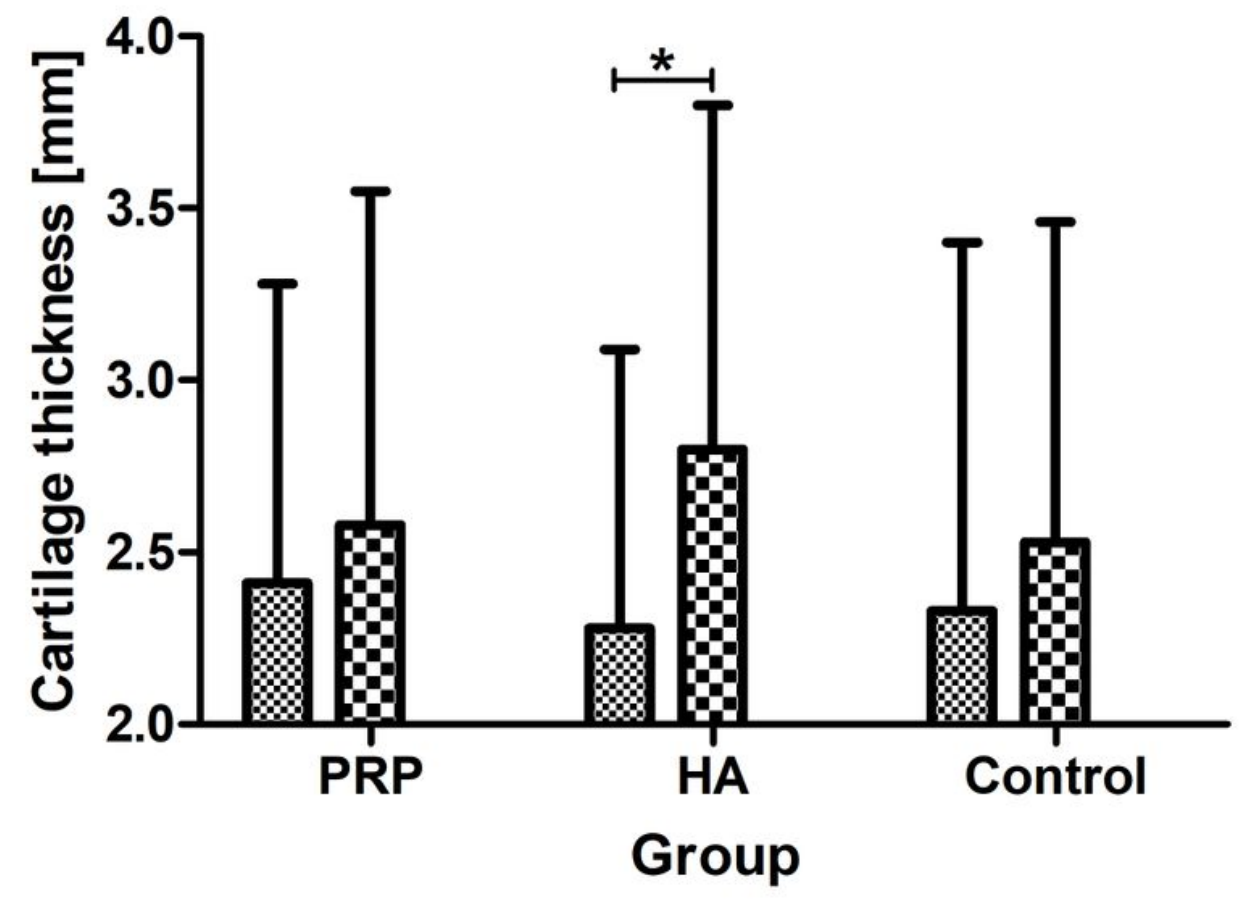

Pre-application Post-application

Figure 4

Comparison of cartilage thickness between groups pre-application and 6 months post-application. Data are presented as mean values $\pm S D$. Statistically significant differences between pre-and post-application were determined using the Wilcoxon signed-rank test ( $\left.{ }^{*} \mathrm{P} \leq 0.05\right)$.

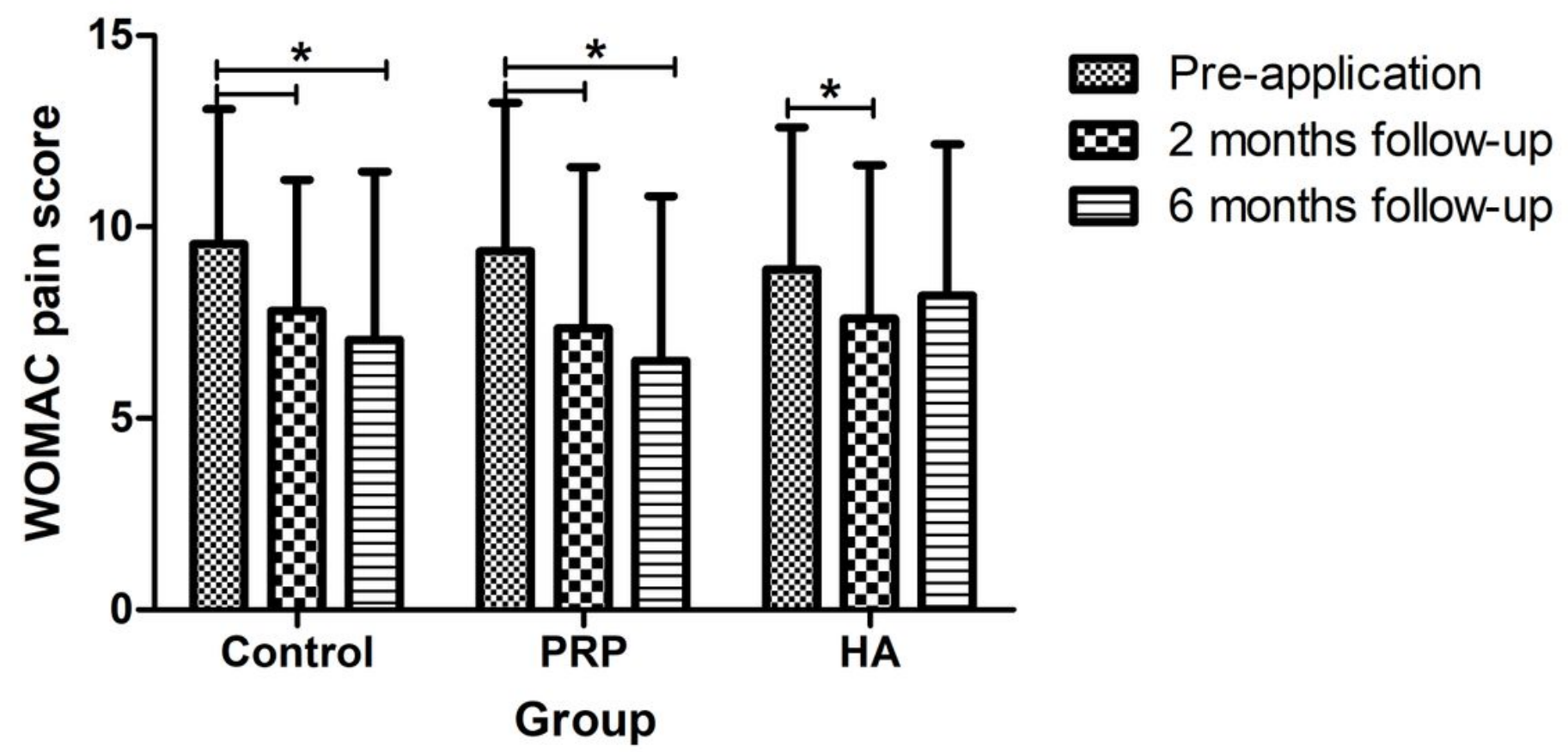


Figure 5

Comparison of pain (WOMAC) between groups at three time points. Data are presented as mean values $\pm S D$. Statistically significant differences between pre- and post-application were determined using the Wilcoxon signed-rank test and in comparison, for control the Mann-Whitney U-test ( $\left.{ }^{\star} P \leq 0.05\right)$.

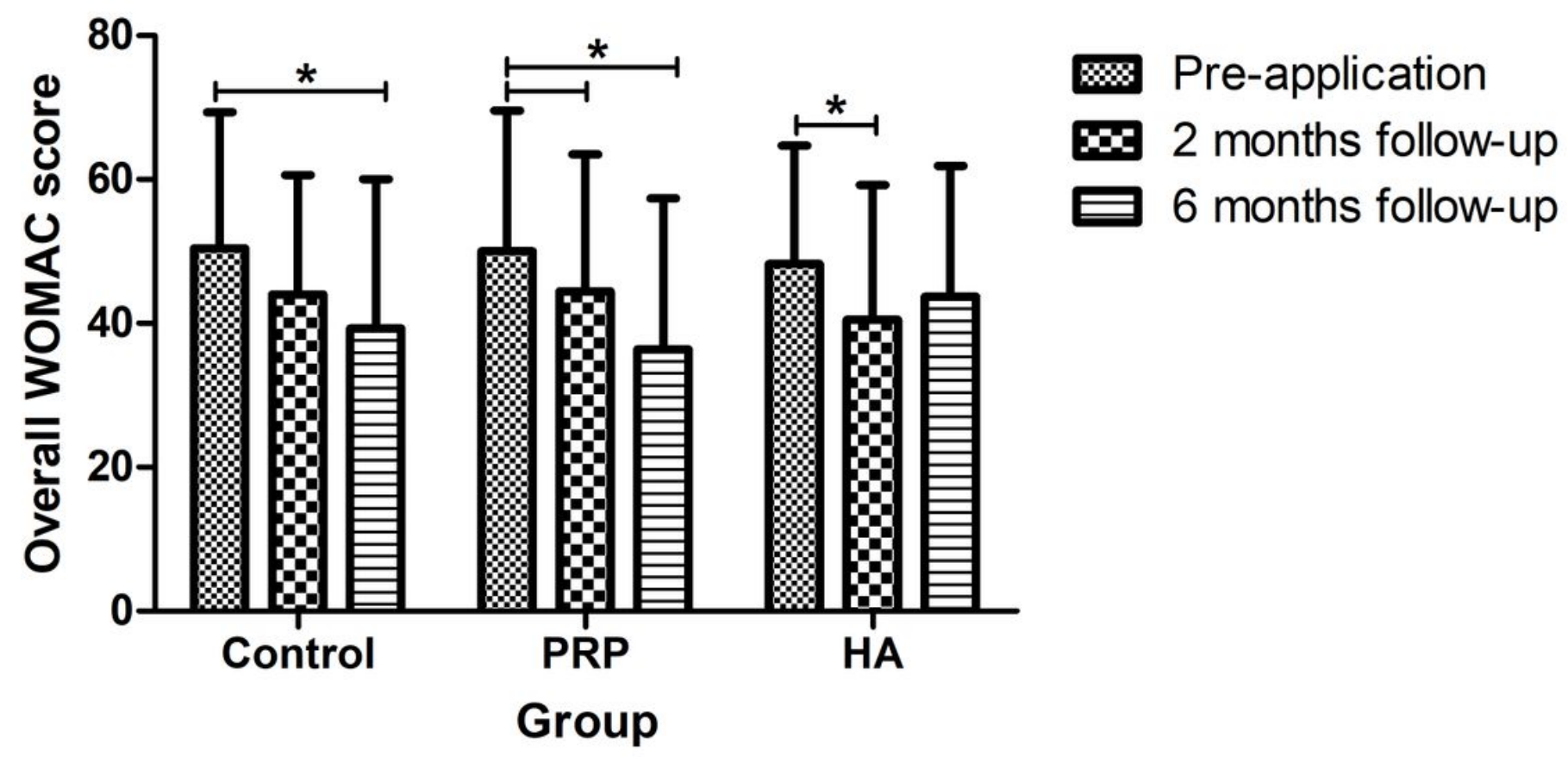

Figure 6

Comparison of WOMAC score between groups at three time points. Data are presented as mean values $\pm S D$. Statistically significant differences between pre- and post-application were determined using Wilcoxon signed-rank test and in comparison, for control the Mann-Whitney U-test ( $\left.{ }^{*} \leq 0.05\right)$. 